'Escuela de Psicología, Facultad de Medicina, Universidad Austral de Chile. Valdivia, Chile. ${ }^{2}$ Instituto Milenio para la Investigación en Depresión y Personalidad (MIDAP). Santiago, Chile.

${ }^{3}$ Centro Interdisciplinario de Estudios del Sistema Nervioso (CISNe), Universidad Austral de Chile. Valdivia, Chile.

${ }^{4}$ Universidad de Almería, España. ${ }^{5}$ Facultad de Ciencias Sociales, Universidad de Chile. Santiago, Chile.

${ }^{6}$ Escuela de Psicología, Pontificia Universidad Católica de Chile. Santiago, Chile. aPsicólogo. bPhD.

Financiamiento Este estudio contó con el apoyo de CONICYT/ PAl, Concurso Nacional Apoyo al Retorno de Investigadores desde el Extranjero, № 82130055 y

del Fondo de Innovación para la Competitividad (FIC) del Ministerio de Economía, Fomento y Turismo, a través de la Iniciativa Científica Milenio, Proyecto IS130005.

Recibido el 5 de diciembre de 2016, aceptado el 26 de abril de 2017.

Correspondencia a: Álvaro I. Langer.

Campus Isla Teja s/n, Valdivia. alvaro.langer@gmail.com

\section{Mindfulness y promoción de la salud mental en adolescentes: efectos de una intervención en el contexto educativo}

\author{
ÁLVARO I. LANGER ${ }^{1,2,3, a, b}$, CARLOS SCHMIDT T,a, \\ JOSÉ MANUEL AGUILAR-PARRA ${ }^{4, \mathrm{a}, \mathrm{b}}$, CRISTIAN CID ${ }^{5, \mathrm{a}}$, \\ ANTONELLA MAGNI ${ }^{6, a}$

\section{Effects of a mindfulness intervention in Chilean high schoolers}

Background: Mindfulness has been conceptualized as paying attention to present moment experience in a non-judgmental manner, and the practice of developing that skill. Aim: To determine the impact of a mindfulness-based intervention on negative emotional states of anxiety, stress, and depression in Chilean high schoolers. Material and Methods: Eighty-eight teenagers aged $13 \pm 0.6$ years ( 46 females) were randomly assigned to a mindfulness group or a control (41 and 47, respectively). The mindfulness intervention consisted in eight weekly 45-minute sessions. A depression, anxiety, and stress scale (DASS-21) was applied at baseline, after the intervention, and at three and six-month follow-up. Results: There was a significant reduction in anxiety, depression, and general symptomatology in the experimental group compared to the control group. However, these changes were not sustained at follow-up. Conclusions: These preliminary results suggest the feasibility and effectiveness of a mindfulness intervention in Chilean schools as a strategy to reduce negative emotional states and prevent risk factors in adolescent population groups.

(Rev Med Chile 2017; 145: 476-482)

Key words: Adolescent; Mental Health; Mindfulness; School Health Services.
L a Organización Mundial de la Salud define la adolescencia como la etapa que transcurre entre los 10 y 19 años, y en la cual se producen cambios que pueden determinar un conjunto de fortalezas para la vida o, por el contrario, pueden aumentar las condiciones de vulnerabilidad social y riesgos para la salud ${ }^{1}$. Esta etapa del desarrollo se caracteriza por cambios fisiológicos, físicos y psicológicos distintivos que ocurren de forma rápida y en la cual pueden aparecer manifestaciones relacionadas con problemas de salud mental, como trastornos de ansiedad y depresivos $^{2}$. Los adolescentes también enfrentan múltiples demandas de adaptación y de rendi- miento en el ámbito familiar, social y académico, las que pueden ser vividas con estrés. En esta etapa se ha evidenciado que existe una fuerte asociación entre estrés y desajuste emocional, en particular, con trastornos internalizantes, como ansiedad, depresión y baja autoestima, y con trastornos externalizantes, como conductas de hostilidad hacia el entorno escolar y dificultades en las relaciones interpersonales ${ }^{3}$.

Los establecimientos educacionales son un lugar en el cual los adolescentes pasan la mayor parte del día, convirtiéndose en un contexto natural en el que se pueden implementar estrategias psicoterapéuticas no tradicionales enfocadas en la 
prevención de problemas psicológicos ${ }^{4}$. De esta manera, el rol de la escuela no sólo se entiende desde la educación formal en donde su función es estimular el desarrollo cognitivo, sino también como una institución que debe promover activamente el desarrollo personal y afectivo, así como el bienestar de todos los estudiantes ${ }^{5}$.

Dentro de las estrategias de intervención que han logrado mayor interés científico en el último tiempo, se encuentran aquellas basadas en mindfulness ${ }^{6,7}$. Se define mindfulness como una forma de conciencia no evaluativa, centrada en el presente, en la que cada pensamiento, sentimiento o sensación que surge en el campo atencional es reconocida y aceptada tal como es, sin juicios de valor $^{6,8,9}$. En un estado de mindfulness, los pensamientos y sentimientos se observan como eventos mentales, sin sobre-identificarse, ni reaccionar ante ellos de forma automática, evitando patrones habituales de reactividad emocional ${ }^{8}$. Entre los mecanismos de acción de mindfulness, se destaca un trabajo sinérgico entre la regulación de la atención, la conciencia en el cuerpo, la regulación emocional y los cambios en las perspectivas del Self(flexibilidad psicológica), que en su conjunto constituyen un proceso de aumento de la autorregulación ${ }^{10,11}$.

Las intervenciones basadas en mindfulness aplicadas en contextos educativos cuentan con un amplio respaldo empírico en la prevención de problemas de salud mental y en la promoción del bienestar psicológico en población infanto-juvenil $^{12-16}$. Específicamente, las intervenciones basadas en mindfulness aplicadas en jóvenes han mostrado reducción de síntomas depresivos ${ }^{17-19}$, síntomas de ansiedad ${ }^{20-23}$ y síntomas de estrés ${ }^{24}$, así como aumento de la autoestima ${ }^{25,26}$. Como antecedente, una reciente revisión de 28 intervenciones basadas en mindfulness para niños y jóvenes en las escuelas reportó un aumento en atributos psicosociales que promueven la conducta prosocial, tales como: involucramiento en el aula, regulación emocional, habilidades sociales, competencia socio-emocional, afrontamiento (coping) de situaciones adversas, afecto positivo, optimismo y mejor conducta en el aula ${ }^{27}$. A pesar de que la investigación y los hallazgos apuntan a la efectividad de las intervenciones basadas en mindfulness, un aspecto relevante a considerar es que no existe mayor información que dé cuenta de los efectos diferenciados entre hombres y mu- jeres. Al respecto, un estudio reciente que abordó las diferencias de sexo, encontró que las mujeres reportaron menos estrés después de la intervención y estaban más comprometidas que los hombres en el taller ${ }^{28}$. En contraste, una revisión sistemática de intervenciones que implementaron mindfulness en contexto escolar, dan cuenta de efectos positivos sobre variables emocionales en adolescentes de ambos sexos, sin embargo, existen ciertas limitaciones metodológicas como el uso de muestras homogéneas que se deben controlar en futuros estudios ${ }^{14}$. En lo que respecta a esta investigación, interesa evaluar si los efectos de mindfulness son diferenciados en la población local dependiendo del sexo.

Si bien existe evidencia empírica sobre los efectos beneficiosos de las intervenciones mindfulness en adolescentes, en Chile no se cuenta con datos empíricos que confirmen la efectividad y factibilidad de su aplicación en el contexto local. En este sentido, contar con estrategias validadas empíricamente para la prevención de problemas de salud mental en las escuelas, permite en parte, brindar soluciones a lo que son las prioridades de salud adolescente tanto a nivel local ${ }^{29}$ como internacional ${ }^{30}$. Específicamente, los objetivos de este estudio son: a) determinar el efecto de mindfulness aplicado en el contexto escolar en la disminución de estados emocionales negativos en población adolescente; b) evaluar si los resultados se mantienen a los tres y seis meses de seguimiento, y c) determinar si la intervención de mindfulness tiene el mismo efecto en hombres que en mujeres. Se espera que los estudiantes disminuyan estados emocionales negativos de depresión, ansiedad y estrés; que los cambios logrados se mantengan en el tiempo, y que no existan diferencias por sexo en los resultados.

\section{Material y Método}

\section{Participantes}

Participaron en el presente estudio un total de 88 alumnos ( 42 hombres y 46 mujeres) pertenecientes a cuatro cursos de $8^{\circ}$ año básico de dos colegios de la ciudad de Santiago, Chile. La edad osciló entre los 12 y los 14 años $(\mathrm{M}=13,37$; $\mathrm{SD}=0,57)$. De los alumnos participantes, 41 asistieron al grupo de mindfulness y 47 al grupo control (lista de espera). Los criterios de exclusión 
fueron la no aceptación del consentimiento informado para participar en el estudio por parte del apoderado y/o la no aceptación del asentimiento informado por parte del alumno. El estudio está basado en los principios éticos de la Declaración de Helsinki y fue aprobado por el comité de ética de la Escuela de Psicología de la Pontificia Universidad Católica de Chile.

\section{Diseño}

Se utilizó un diseño experimental, controlado aleatorizado, con evaluación pre y post intervención, y seguimiento a los 3 y 6 meses. El taller se desarrolló en 8 sesiones de 45 min cada una, una vez por semana durante dos meses consecutivos. Se utilizó metodología cuantitativa para evaluar la eficacia del ensayo controlado aleatorizado.

\section{Instrumentos}

Escalas de Depresión Ansiedad y Estrés (DASS21). Es una escala de auto-reporte dimensional que fue diseñada para medir los estados emocionales negativos de depresión, ansiedad y estrés. A los participantes se les solicita que respondan cada item de acuerdo a la presencia e intensidad de cada síntoma en la última semana. La versión chilena fue validada en adolescentes estudiantes de enseñanza media ${ }^{31}$, observándose buenas características psicométricas.

\section{Procedimiento}

En cada colegio donde fue aplicado el estudio, un curso fue asignado al grupo experimental y otro curso al grupo control. En el grupo experimental se implementó una intervención basada en mindfulness. Específicamente, se realizó una breve sesión introductoria sobre la temática del taller y sus principales aplicaciones y posteriormente se realizaron 8 sesiones semanales de 45 min cada una, durante el horario de clases de los participantes. Se aplicó el currículum de mindfulness para adolescentes del programa Mindfulness in Schools Project (MiSP) ${ }^{32}$ específicamente desarrollado para jóvenes entre 11 y 18 años. Éste fue diseñado tanto del Mindfulness-Based Stress Reduction (MBSR) ${ }^{6,33}$ como del Mindfulness-Based Cognitive Therapy $(\mathrm{MBCT})^{34}$, el cual se encuentra adaptado para población adolescente y para ser implementado en la sala de clases. Cada sesión, al igual que MBSR, tenía un eje temático en torno al cual se desarrollaba la lección. Asimismo, se utilizaron diversos ejercicios de mindfulness, tales como: escáner corporal, meditación sentada, meditación del caminar, entre otros. A cada participante se le entregó un cuadernillo con el resumen de cada lección y los respectivos ejercicios que debían realizarse en casa. Estos ejercicios se dividieron en prácticas formales, por ejemplo, realizar el escáner corporal y prácticas informales, las cuales buscan llevar mindfulness a las actividades cotidianas, por ejemplo, al lavarse los dientes. Además, se entregó un audio que contenía las principales meditaciones. Todas las sesiones fueron realizadas por un psicólogo certificado en el currículum mindfulness.

\section{Análisis de datos}

En primer lugar, se realizó un MANOVA para descartar diferencias de partida en pretest en los grupos, en relación a edad y género. Posteriormente, con el objetivo de conocer posibles diferencias entre los grupos control y experimental, se realizó una comparación en el pretest, postest y el seguimiento a los tres y seis meses de las puntuaciones medias en DASS 21, complementadas por las puntuaciones Delta y el tamaño del efecto $(d$ de Cohen) a través de las pruebas $t$ de student. Para valorar los efectos del programa (diferencias intra-grupo) se calculó un ANOVA de medidas repetidas con medidas pretest, postest, y seguimiento a los 3 y 6 meses, acompañado del tamaño del efecto a través de $\eta 2$ y de la comparación por pares de Bonferroni. Finalmente se realizó un ANCOVA para verificar si el género del estudiante influyó en sus puntuaciones y por tanto, si el programa tiene el mismo efecto en hombres que en mujeres. Todos los análisis fueron realizados a través del paquete estadístico SPSS.

\section{Resultados}

El análisis de la diferencia de medias pretest entre los grupos control y experimental, tal y como se puede apreciar en la Tabla 1, no reflejó diferencias estadísticamente significativas entre ellos en ninguna de las variables analizadas. En relación al postest, tampoco se observaron diferencias estadísticamente significativas entre ellos, pero se observa que el tamaño del efecto es casi moderado en relación a depresión y ansiedad, lo que podría sugerir que el efecto del tratamiento sobre esa variable sí difiere entre ambos grupos. $\mathrm{Si}$ atendemos a las puntuaciones medias, se aprecia 
que el grupo experimental disminuyó sus niveles de depresión y ansiedad respecto al grupo control, el que empeoró ligeramente dichos niveles. Cuando se valora la diferencia entre los dos grupos en pre y postest a través de las puntuaciones delta, se confirma lo que apuntaba el tamaño del efecto en la prueba t: existen diferencias estadísticamente significativas entre el grupo control y experimental en depresión, ansiedad y en la puntuación total del DASS, con los resultados anteriormente expuestos.

En la Tabla 2, no se observaron diferencias significativas en el seguimiento a los tres y seis meses. Sin embargo, al atender a las diferencias a través de las puntuaciones delta, se observan efectos casi moderados en relación a la ansiedad en el seguimiento a los tres meses y en depresión, en el seguimiento a los seis meses.

Con el fin de conocer los cambios producidos (intra-grupo) en relación al grupo experimental en los diferentes momentos pretest, postest y seguimiento a los 3 y 6 meses, los datos obtenidos fueron sometidos a la prueba ANOVA de medidas repetidas (Tabla 3). Se encontraron diferencias estadísticamente significativas en todos los fac- tores del DASS-21: ansiedad, depresión, estrés y DASS puntuación total en relación a las diferentes mediciones realizadas. En todas ellas, el tamaño del efecto calculado a través de $\eta 2$ muestra que la efectividad del tratamiento sobre estas variables es grande. Al aplicar la comparación por pares de Bonferroni, se aprecia que la diferencia más amplia se recoge entre el pretest y el seguimiento a los seis meses, tal y como se puede apreciar en la Tabla 3. Se observan descensos importantes de depresión, ansiedad y estrés.

Finalmente, y con el objetivo de comprobar si las mejoras observadas tras la aplicación del programa en depresión, ansiedad y estrés, son independientes del género, se realiza un análisis de covarianza (ANCOVA), no encontrándose diferencias entre estudiantes hombres y mujeres en las variables del DASS-21 (ansiedad, estrés y depresión), en ninguna de las comparaciones pretest-postest que puedan atribuirse al género $[\mathrm{F}(3$, $34)=0,981, p=0,413, \eta 2=0,080)$, pretest-seguimiento 3 meses $[\mathrm{F}(4,22)=0,427, \mathrm{p}=0,788$, $\eta 2=0,072]$ y pretest-seguimiento 6 meses $[\mathrm{F}(4$, 18) $=0,481, p=0,750, \eta 2=0,096]$.

Tabla 1. Comparación diferencias de medias entre el grupo de mindfulness y el grupo control en variables sintomáticas, pre y post intervención

\begin{tabular}{|c|c|c|c|c|c|c|c|c|c|c|c|}
\hline \multirow[b]{4}{*}{ Depresión } & \multicolumn{2}{|c|}{ Mindfulness } & \multirow{3}{*}{$\begin{array}{l}\text { Grupo } \\
\text { Pretest } n \\
=47 \\
\text { M (SD) }\end{array}$} & \multirow{3}{*}{$\begin{array}{l}\text { Control } \\
\text { Postest } \\
n=47 \\
M \text { (SD) }\end{array}$} & \multicolumn{7}{|c|}{ Diferencias intergrupos } \\
\hline & \multirow{2}{*}{$\begin{array}{l}\text { Pretest } \\
n=41 \\
M \text { (SD) }\end{array}$} & \multirow{2}{*}{$\begin{array}{l}\text { Postest } \\
n=41 \\
M \text { (SD) }\end{array}$} & & & \multicolumn{2}{|c|}{ Pre } & \multicolumn{3}{|c|}{ Post } & \multicolumn{2}{|c|}{$\begin{array}{c}\text { Delta } \\
\text { post-pre }\end{array}$} \\
\hline & & & & & $\mathbf{t}$ & $\mathbf{p}$ & $\mathbf{T}$ & $\mathbf{p}$ & d & $\mathbf{t}$ & $\mathbf{p}$ \\
\hline & $11,58 \quad(7,53)$ & 8,24 & $0,06(5,84)$ & 1 & $-1,047$ & 0,298 & 1,702 & 0,092 & $-0,365$ & 2,045 & 60 \\
\hline Ansiedad & (J), (0, (0, & $9,46 \quad(7,87)$ & $10,74(6,51)$ & $13,24 \quad(8,53)$ & $-1,527$ & 0,131 & 1,334 & 0,185 & -0 & 1,964 & 0,000 \\
\hline (3) & 然 & $7,75 \quad(7,1$ & &, $04 \quad(7$ & $-1,497$ & 0,140 & 4 & 0 & -( & 1,723 & 0,08 \\
\hline DASS total & $17,78(11,35)$ & $12,73(10,31)$ & $14,68(8,01)$ & $15,74(10,73)$ & $-1,459$ & 0,149 & 1,338 & 0,185 & $-0,286$ & 2,015 & 0,047 \\
\hline
\end{tabular}

Tabla 2. Comparación de las diferencias entre medias entre el grupo de mindfulness y el grupo control en variables sintomáticas a través del seguimiento a los tres y seis meses después de finalizar el taller

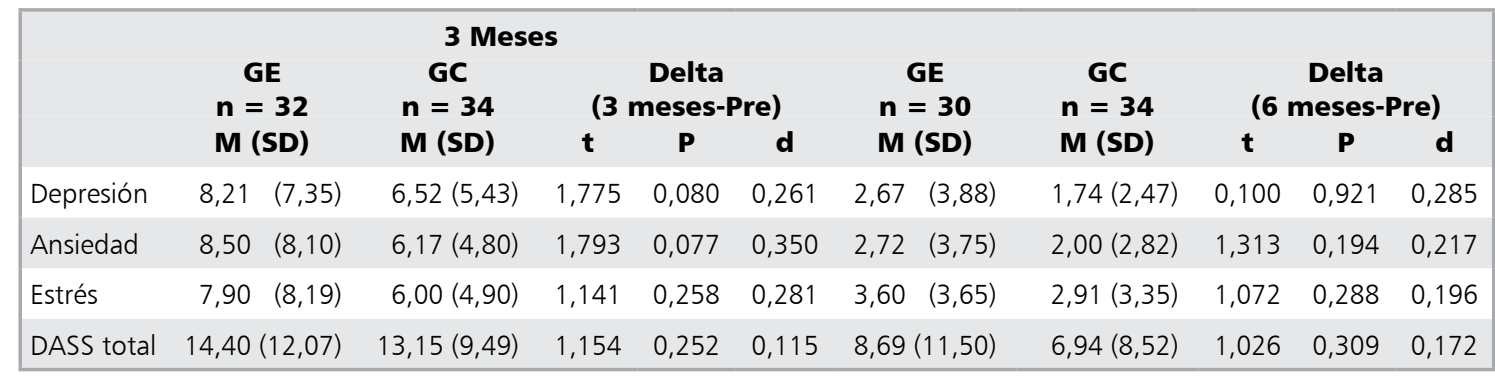


Tabla 3. Comparación de la diferencias entre medias intragrupo mindfulness en variables sintomáticas pre, post intervención, seguimiento tres y seis meses

\begin{tabular}{|c|c|c|c|c|c|c|c|c|}
\hline $\begin{array}{l}\text { Instru- } \\
\text { mento } \\
\text { DASS-21 }\end{array}$ & $\begin{array}{l}\text { M (SD) } \\
\text { Pretest }\end{array}$ & $\begin{array}{l}\text { M (SD) } \\
\text { Postest }\end{array}$ & $\begin{array}{l}\text { M (SD) } \\
\text { Segto } 3\end{array}$ & $\begin{array}{l}\text { M (SD) } \\
\text { Segto } 6\end{array}$ & $\mathbf{F}$ & Sig. & $\eta^{2}$ & $\begin{array}{l}\text { Comparación } \\
\text { post-hoc }\end{array}$ \\
\hline Depresión & $11,58(7,53)$ & $8,24(6,28)$ & $8,21(7,35)$ & $2,67(3,88)$ & 21,121 & 0,000 & 0,771 & $\begin{array}{l}\text { Pretest-Seguimiento } 6^{* * *} \\
\text { Postest-Seguimiento } 6^{* *} \\
\text { Seguimiento } 3 y \\
\text { Seguimiento } 6^{* * *}\end{array}$ \\
\hline Ansiedad & $13,24(8,53)$ & $9,46(7,87)$ & $8,50(8,10)$ & $2,72(3,75)$ & 20,203 & 0,000 & 0,700 & $\begin{array}{l}\text { Pretest-Seguimiento } 3^{*} \\
\text { Pretest-Seguimiento } 6^{* * *} \\
\text { Postest-Seguimiento } 6^{* *} \\
\text { Seguimiento } 3 y \\
\text { Seguimiento } 6^{* * *}\end{array}$ \\
\hline Estrés & $10,73(7,80)$ & $7,75(7,17)$ & $7,90(8,19)$ & $3,60(3,65)$ & 10,296 & 0,000 & 0,553 & $\begin{array}{l}\text { Pretest-Seguimiento } 6^{* * *} \\
\text { Seguimiento } 3 y \\
\text { Seguimiento } 6^{*}\end{array}$ \\
\hline DASS total & $17,78(11,35)$ & $12,73(10,31)$ & $14,40(12,07)$ & $8,69(11,50)$ & 3,371 & 0,038 & 0,325 & Pretest-Seguimiento 6 * \\
\hline
\end{tabular}

\section{Discusión}

El presente estudio piloto se enfocó en determinar el efecto de una intervención basada en mindfulness en la disminución de estados emocionales negativos en población adolescente escolarizada de la ciudad de Santiago, Chile. En esta línea, los resultados confirman parcialmente nuestra hipótesis, ya que el grupo experimental, en comparación al control, demostró una disminución estadísticamente significativa en sintomatología ansiosa y depresiva, como también en el malestar psicológico general (evaluado a través de la puntación global de la escala DAAS). Estos hallazgos son coherentes con estudios previos que han demostrado que mindfulness aplicado en contextos educativos produce una reducción significativa de síntomas de ansiedad ${ }^{21,35}$ y depresión ${ }^{19}$.

Asimismo, se realizó un seguimiento a los tres y seis meses post intervención, encontrándose que no se mantuvieron los cambios en ambos períodos. En contraste, estudios previos demuestran que los efectos se mantienen hasta tres meses post intervención, sin embargo, estas diferencias encontradas se encuentran supeditadas a que los participantes continúen con la práctica una vez finalizada la intervención. Por ejemplo, Kuyken et al. $^{18}$, demostraron que la mantención de los efectos a los tres meses, tenía relación con que los alumnos seguían practicando fuera del colegio. Asimismo, Biegel, Brown, Shapiro \& Schubert ${ }^{21}$ mostraron una mejoría en síntomas de ansiedad y depresión en jóvenes que siguieron practicando meditación mindfulness transcurrido 3 meses de terminada la intervención, en comparación con quienes no lo hicieron.

Interesantemente, al analizar los cambios intrasujetos en el grupo mindfulness respecto a los síntomas de depresión, ansiedad y estrés, estos sí mostraron una disminución significativa a los tres y seis meses de seguimiento. Sin embargo, estos datos deben ser evaluados con cautela, pues al recogerse en un contexto natural, no se tiene control de otros factores que pueden influir en el cambio de ánimo de los adolescentes luego de la intervención, especialmente si se considera que la adolescencia es una etapa evolutiva compleja, marcada por grandes cambios y por una gran vulnerabilidad a los factores contextuales del desarrollo ${ }^{31}$.

Entre los aspectos que podrían explicar las diferencias encontradas con estudios previos se encuentra la forma de aleatorización de la muestra, ya que en el presente estudio se optó por aleatorizar cursos de un mismo nivel educativo (e.g. cursos de $8^{\circ}$ Básico), en contraste con estudios que han realizado aleatorización a nivel de individuos o de los centros educativos 5 . Por otro lado, el tamaño 
de la muestra fue relativamente pequeño y el uso de un grupo control activo habría sido el diseño ideal a utilizar en este tipo de estudios ${ }^{14}$. Además, no se controló si los adolescentes mantuvieron la práctica luego de finalizado el taller. Ésta es una limitación considerable, ya que como se mencionó, la mantención de los efectos está relacionado con seguir practicando meditación mindfulness.

Por otro lado, no se encontraron diferencias de género en los resultados, lo que da cuenta del beneficio de la meditación mindfulness para ambos sexos, al menos en la reducción de síntomas de depresión y ansiedad en población escolar chilena.

Hasta donde tenemos conocimiento, éste es el primer estudio en Chile y Sudamérica que aplica una intervención basada en mindfulness en el contexto educativo bajo un diseño aleatorizado y controlado. En este sentido, entre los aspectos relevantes de incluir mindfulness en contextos escolares están el promover habilidades y entregar herramientas de regulación emocional y conductual que permitan a los adolescentes gestionar de mejor forma sus estados emocionales y afrontar las múltiples demandas del entorno escolar que pueden generar desajustes emocionales en adolescentes vulnerables ${ }^{3}$. En este sentido, una de las habilidades que se promueven a través del entrenamiento de mindfulness, es la capacidad de observar y prestar atención a los pensamientos y estados de ánimo, los cuales son elementos esenciales para la auto-regulación de los adolescentes.

Consideramos que los resultados obtenidos son relevantes debido a que proporcionan resultados preliminares sobre la viabilidad y efectividad de la implementación de mindfulness en el contexto educacional chileno. En este sentido, los datos reportados en este estudio son el primer paso de un área de investigación que busca generar estrategias de prevención enfocadas en el contexto natural de los jóvenes y, de esta forma, reducir sintomatología depresiva y ansiosa, las cuales tienen un impacto negativo en el rendimiento académico y en el desempeño psicosocial e individual, aumentando el riesgo de otros tipos de problemas de salud mental en los adolescentes, como el abuso de alcohol ${ }^{36,37}$. En efecto, mindfulness puede ser una estrategia para la prevención de problemas psicológicos y disminución de factores de riesgo en población adolescente, las cuales son líneas de trabajo prioritarias en las políticas de salud tanto a nivel local como internacional. Esto sugiere la relevancia de generar futuras investigaciones que, por un lado, superen las limitaciones presentadas en este estudio y, por otro, exploren la relación de mindfulness con otras variables más allá de las sintomáticas, como las interpersonales y sociales desde una lógica que considere los marcos socio-culturales locales.

\section{Referencias}

1. Ministerio de Salud de Chile (MINSAL). Guía práctica de Consejería para Adolescentes y Jóvenes [Internet]. Santiago, Chile; 2011. Disponible en: http://web.minsal.cl/portal/ url/item/aaa27720f365a745e04001011e011120.pdf

2. Costello EJ, Mustillo S, Erkanli A, Keeler G, Angold A.. Prevalence and Development of Psychiatric disorders in Childhood and Adolescence. Arch Gen Psychiatry 2003; 60: 837-44.

3. Escobar Espejo M, Blanca MJ, Fernández-Baena FJ, Trianes Torres MV. Adaptación española de la escala de manifestaciones de estrés del Student Stress Inventory (SSI-SM). Psicothema 2011; 23 (3): 475-85.

4. Wisner B, Jones B, Gwin D. School Based meditation practices for adolescents: a resource for strengthening self regulation, emotional coping and self-esteem. Children and Schools 2010; 32 (3): 150-9.

5. Zenner C, Herrnleben-Kurz S, Walach H. Mindfulness-based interventions in schools-a systematic review and meta-analysis. Front Psychol 2014; 5: 603.

6. Kabat-Zinn J. Full catastrophe living: Using the wisdom of your mind to face stress, pain and illness. New York: Dell; 1990.

7. Segal Z, Williams J, Teasdale J. Mindfulness based cognitive therapy for depression. New York, NY: Guilford; 2002.

8. Bishop SR, Lau M, Shapiro S, Carlson L, Anderson ND, Carmody J, et al. Mindfulness: a proposed operational definition. Clin Psychol Sci Pract 2004; 11: 230-41.

9. Kabat-Zinn J. Meditation. In J. C. Holland (Ed.), Psycho-oncology (pp. 767-79). New York: Oxford University Press. 1998.

10. Hölzel BK, Lazar SW, Gard T, Schuman-Olivier Z, Vago DR, Ott U. How Does Mindfulness Meditation Work? Proposing Mechanisms of Action from a Conceptual and Neural Perspective. Perspect Psychol Sci 2011; 6 (6): 537-9.

11. Tang YY, Hölzel BK, Posner MI. The neuroscience of mindfulness meditation. Nat Rev Neurosci 2015; 16: 213-25.

12. Burke CA. Mindfulness-based approaches with children and adolescents: A preliminary review of current research in an emergent field. J Child Fam Stud 2010; 19: 133-44. 
13. Harnett PH, Dawe S. Review: The contribution of mindfulness-based therapies for children and families and proposed conceptual integration. Child Adolesc Ment Health 2012; 17 (4): 195-208.

14. Langer A, Ulloa V, Cangas A, Rojas G, Krause M. Mindfulness-based interventions in secondary education: a qualitative systematic review/Intervenciones basadas en mindfulness en educación secundaria: una revisión sistemática cualitativa. Estudios de Psicología 2015; 36: 533-70.

15. Lerma-Cabrera J, Steinebach P, Carvajal F, Ulloa V, Cid-Parra C, Langer A. Factores de riesgo asociados al consumo problemático de alcohol en la adolescencia: El rol preventivo de mindfulness. Psy, Soc, \& Educ 2015; 7 (1): 57-69.

16. Parker AE, Kupersmidt JB, Mathis ET, Scull TM, Sims C. The impact of mindfulness education on elementary school students: evaluation of the Master Mind program. Adv Sch Ment Health Promot 2014; 7 (3): 184-204.

17. Ames CS, Richardson J, Payne S, Smith P, Leigh E. Mindfulness based cognitive therapy for depression in adolescents. Child Adolesc Ment Health 2014; 19 (1): 74-8.

18. Kuyken W, Weare K, Ukoumunne OC, Vicary R, Motton N, Burnett R, et al. Effectiveness of the mindfulness in schools programme: Non-randomised controlled feasibility study. Br J Psychiatry 2013; 203: 126-31.

19. Raes F, Griffith J, Van der Gucht K, Williams J. School-based prevention and reduction of depression in adolescents: A cluster-randomized controlled trial of a mindfulness group program. Mindfulness 2014; 5: 477-86.

20. Beauchemin J, Hutchins T, Patterson F. Mindfulness meditation may lessen anxiety, promote social skills, and improve academic performance among adolescents with learning disabilities. Complement Health Pract Rev 2008; 13: 34-45.

21. Biegel GM, Brown KW, Shapiro SL, Schubert CM. Mindfulness-based Stress Reduction for the treatment of adolescent psychiatric outpatients: a randomized clinical trial. J Consult Clin Psychol 2009; 77 (5): 855-66.

22. Franco C, Mañas I, Cangas AJ, Gallego J. Exploring the effects of a mindfulness program for students of secondary school. International Journal of Knowledge Society Research 2011; 2: 14-28.

23. Ramos N, Hernández S, Blanca M. Efecto de un programa integrado de mindfulness e inteligencia emocional sobre las estrategias cognitivas de regulación emocional. Ansiedad y Estrés 2009; 15 (2-3): 207-16.

24. Broderick P, Metz S. Learning to breathe: A pilot trial of a mindfulness curriculum for adolescents. Adv Sch
Ment Health Promot 2009; 2: 35-46.

25. Franco C, de la Fuente M, Salvador M. Impacto de un programa de entrenamiento en conciencia plena (mindfulness) en las medidas del crecimiento y la autorrealización personal. Psicothema 2011; 23: 58-65.

26. Soriano E, Franco C. Mejora de la autoestima y de la competencia emocional en adolescentes inmigrantes sudamericanos residentes en España a través de un programa psicoeducativo de Mindfulness (conciencia plena). Revista de Investigación Educativa 2010; 28: 297-312.

27. Felver J, Clis-de Hoyos C, Tezanos K, Singh N. A systematic review of mindfulness-based interventions for youth in school settings. Mindfulness 2016; 7: 3445.

28. Bluth K, Roberson PNE, Girdler SSJ. Adolescent Sex Differences in Response to a Mindfulness Intervention: A Call for Research. Child Fam Stud (2017). doi:10.1007/ s10826-017-0696-6.

29. Ministerio de Salud de Chile (MINSAL). Programa nacional de salud integral de adolescentes y jóvenes [Internet]. Santiago, Chile; 2012. Disponible en: ttp://web.minsal.cl/ portal/url/item/d263acb5826c2826e04001016401271e.pdf.

30. Organización Panamericana de Salud. Estrategia y plan de acción regional sobre los adolescentes y jóvenes. Washington, DC: OPS; 2009. Disponible en: http://new.paho.org/ hq/dmdocuments/2011/Estrategia-y-Plan-de-Accion-Regional-sobre-los-Adolescentes-y-Jovenes.pdf.

31. Román F, Vinet E, Alarcón A. Escalas de Depresión, Ansiedad y Estrés (DASS-21): Adaptación y propiedades psicométricas en estudiantes secundarios de Temuco. Revista Argentina de Clínica Psicológica XXIII 2014; 179-90.

32. Mindfulness in Schools Project (MiSP) [Internet]. England: Mindfulness in Schools Project [citado el 30 de marzo de 2017]. Disponible en: http://mindfulnessinschools.org/

33. Kabat-Zinn J. Mindfulness-based interventions in context: Past, present, and future. Clinical Psychology: Science and Practice 2003; 10 (2): 144-56.

34. Segal Z, Williams J, Teasdale J. Mindfulness based cognitive therapy for Depression. New York: Guilford; 2002.

35. Hofmann SG, Sawyer AT, Witt AA, Oh D. The effect of mindfulness-based therapy on anxiety and depression: A meta-analytic review. J Consult Clin Psychol 2010; 78: 169-83.

36. Lynch F, Clarke G. Estimating the economic burden of depression in children and adolescents. Am J Prev Med 2006; 31: 143-51.

37. Reardon L, Leen-Feldner E, Hayward C. A critical review of the empirical literature on the relation between anxiety and puberty. Clin Psychol Rev 2009; 29: 1-23. 